\title{
Proposal of a novel method for observing the breast by high-resolution ultrasound imaging: understanding the normal breast structure and its application in an observational method for detecting deviations
}

\author{
Ayumi Izumori · Rie Horii · Futoshi Akiyama • \\ Takuji Iwase
}

Received: 18 July 2011/Accepted: 18 October 2011/Published online: 29 November 2011

(C) The Japanese Breast Cancer Society 2011

\begin{abstract}
Background With the recent advances in modalities for diagnostic imaging of the breast, it is now essential to detect isoechoic masses and small nonmass lesions, to which little attention has so far been paid using ultrasound (US) of the breast. It will be possible with the observation method to understand normal breast structural images and anatomy. We elucidated the detailed histological architecture of the normal breast, information indispensable for diagnostic US of the breast.

Methods Verification of the above hypotheses was carried out using the breasts of 11 patients who underwent total mastectomy at our clinic.

Results Isoechoic structures with fat are lobules, all ducts, and surrounding stroma that support the ducts; intervening hyperechoic areas are edematous stroma and fat-containing stroma that support the breast. By taking an isoechoic structure that reflects the course of the ducts as the basic structure for observation, the boundary between the lobes can be inferred.

Conclusions Detection of "deviations from the normal structure" using the "method for interpreting threedimensional ultrasound images of mammary lobes" is a radical new approach for diagnosing breast cancer. This technique is very simple and amenable to standardization once one understands the underlying theory. Furthermore, it is useful as a screening method as well as for easy
\end{abstract}

A. Izumori $(\bowtie) \cdot$ R. Horii · F. Akiyama - T. Iwase

The Cancer Institute Hospital of the Japanese Foundation

for Cancer Research, 3-8-31 Ariake, Koto-ku,

Tokyo 135-8550, Japan

e-mail: izuiso@mail2.netwave.or.jp detection of faint minute lesions that can only be detected by magnetic resonance imaging or second-look targeted US.

Keywords Ultrasonography · High-resolution ·

Diagnosis · Three-dimensional - Histological architecture

\section{Introduction}

With the recent advances in modalities for diagnostic imaging of the breast, magnetic resonance imaging (MRI) is now widely used for breast examination. MRI is highly sensitive and is frequently used for the screening of patients at high risk of breast cancer and for the diagnosis of the spread of the tumor within the tumor-bearing breast. However, differentiation of positive findings between benign and malignant disease raises a problem because physiological changes and benign lesions also show strong enhancement. Currently, in the US and Europe, MRI-guided needle biopsy is performed as a method for detailed examination, whereas in Japan, Second Look Targeted Ultrasound $\left(2^{\text {nd }}\right.$ LookUS $)$ has become a method of choice for this purpose because it is simpler and can be used in many clinical sites. In performing $2^{\text {nd }}$ LookUS after MRI, it is necessary to detect and identify many similar benign lesions. However, it is impossible to detect and identify these lesions by the traditional method, which is based on the detection of lesions by focusing on hypoechoic areas. Thus, it is now essential to detect isoechoic masses and small nonmass lesions to which little attention has so far been paid. In addition, since US has been used until now to confirm lesions that were detected by palpation or mammography, the method has been used for evaluating only those 
hypoechoic lesions that have grown to a considerable extent. In the current whole breast scan with US, it is necessary to detect isoechoic lesions and small nonmass lesions that are asymptomatic and difficult to visualize by mammography.

In order to achieve these goals, we have focused on the continuity of the normal structure of the breast and utilized an observational method with which we detect lesions on the basis of the "interruption of continuity and disorder" instead of searching for hypoechoic areas as the hallmark of malignant lesions. We believe that this observational method is simple and amenable to standardization once one understands the basic principle. However, it is essential to understand the normal structure of the breast before one can understand the basic principle and actually recognize lesions by using this observational method. Thus, it is critical to correlate the anatomy of the normal tissue and the images of high-resolution US.

Previous reports on the structure of the normal breast described the anatomy of the terminal duct-lobular unit (TDLU) and changes observed during growth and lactations $[1,2]$. With regard to the distribution of the structure of mammary lobes, discussions based on the structure of the ducts are the only information available in the published literature, including information obtained by tracing the ducts dilated during the lactating period using US [3, 4], by using modalities other than US, e.g., infusion of resin into the ducts, and by three-dimensional image processing of the duct specimens [5, 6]. Stavros claims that "the major ducts and surrounding stroma," "terminal ducts," and "lobules" are visualized as isoechoic areas by US [7], but they do not match the actual histological pictures in which the isoechoic structure is larger in diameter.

We have therefore formulated the following three hypotheses on the structure of the normal breast on the basis of our US imaging investigations and clinical experiences. We subjected these hypotheses to verification by correlation between findings obtained from histopathological specimens and high-resolution US images, and attempted to provide a histopathological interpretation of the normal structure observed by high-resolution US.

On the basis of these results, we will explain the basic principle and the observational method for the "method for interpreting three-dimensional ultrasound images of mammary lobes," the method we have been using.

\section{Hypotheses}

We formulated the following hypotheses based on our clinical experience of the "method for interpreting threedimensional ultrasound images of mammary lobes."
Hypothesis 1

When compared with the images of histological specimens, isoechoic structures appear thicker for ducts and larger for lobules. Therefore, the observed isoechoic structure may actually include the ducts and lobules as well as the surrounding structures.

Hypothesis 2

Isoechoic structures are observed even in the breast of elderly people who are considered to have atrophied ducts and lobules, which suggests that there are residual structures surrounding the lobules and ducts.

Hypothesis 3

A sheet structure impenetrable to isoechoic structures is observed within the mammary gland. This may be the boundary between the lobes.

\section{Patients and methods}

Verification of the above hypotheses was performed using the breasts of 11 patients who underwent total mastectomy in our clinic. The ultrasound device used was the Toshiba (Tochigi, Japan) Ultrasound Diagnostic System Aplio XG attached with a probe (PLT-1204BX; center frequency $14 \mathrm{MHz}$ ). One day before the operation, a US image of the apparently normal area was recorded to obtain moving and still ultrasonic images in the radial direction from the nipple. In order to obtain histological specimens with a cut surface at the same site and in the same direction corresponding to the US images taken before the operation, the removed breasts were again subjected to US examination, marked for the site and direction, and then subjected to the preparation of histological specimens. Histological specimens were prepared by cutting the tissue samples into 5- to 7-mm-thick sections and staining with hematoxylin and eosin (HE). By taking into consideration the relative, and not the absolute, grey scale of US images, we examined in detail how the tissue constituents in the HE-stained specimens of each patient were visualized on US images.

\section{Results}

Findings in normal tissues

Images of HE-stained specimens showing the histological structure of the normal mammary gland are provided 
A

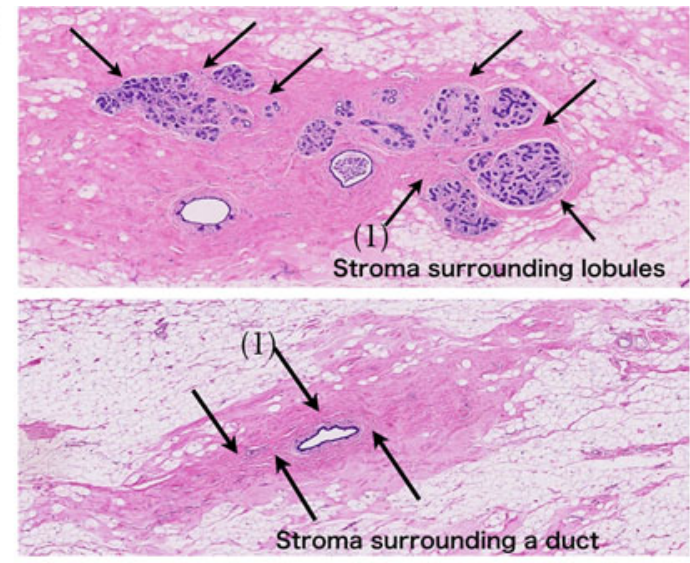

B

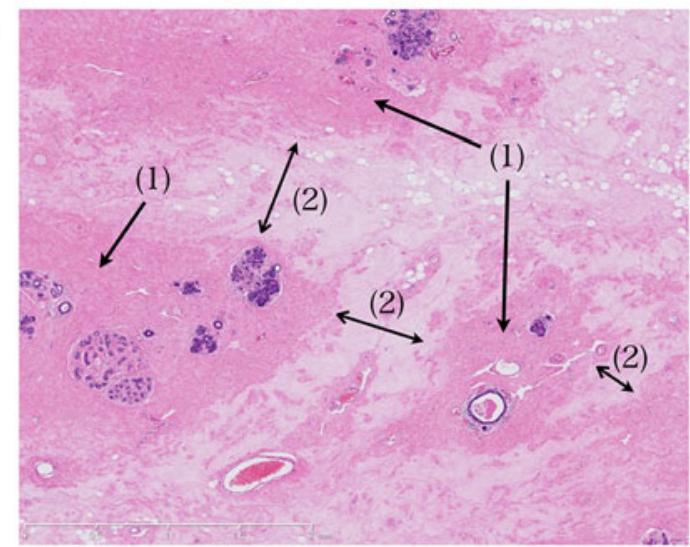

C
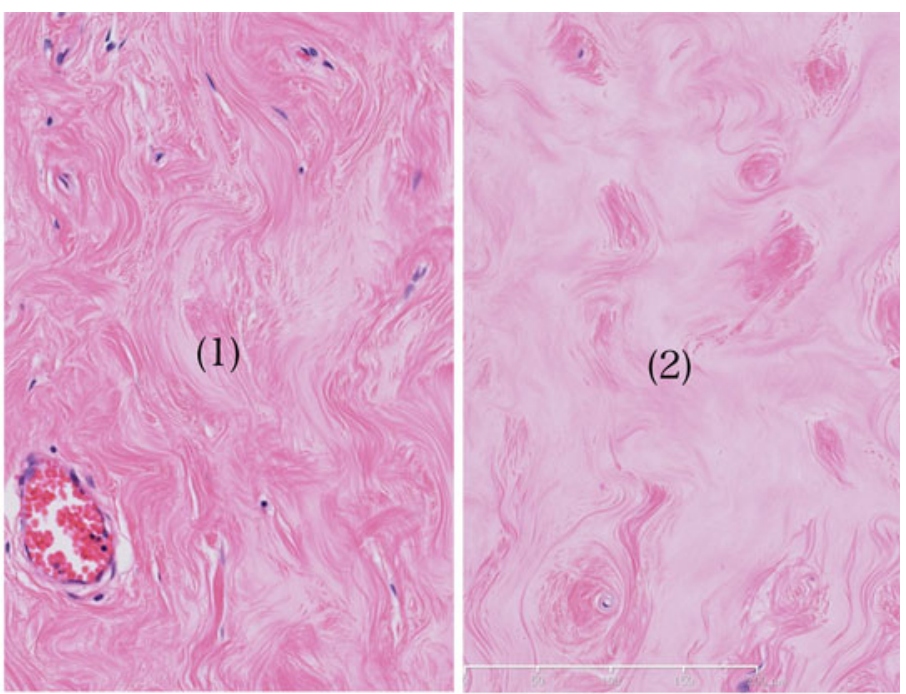

D
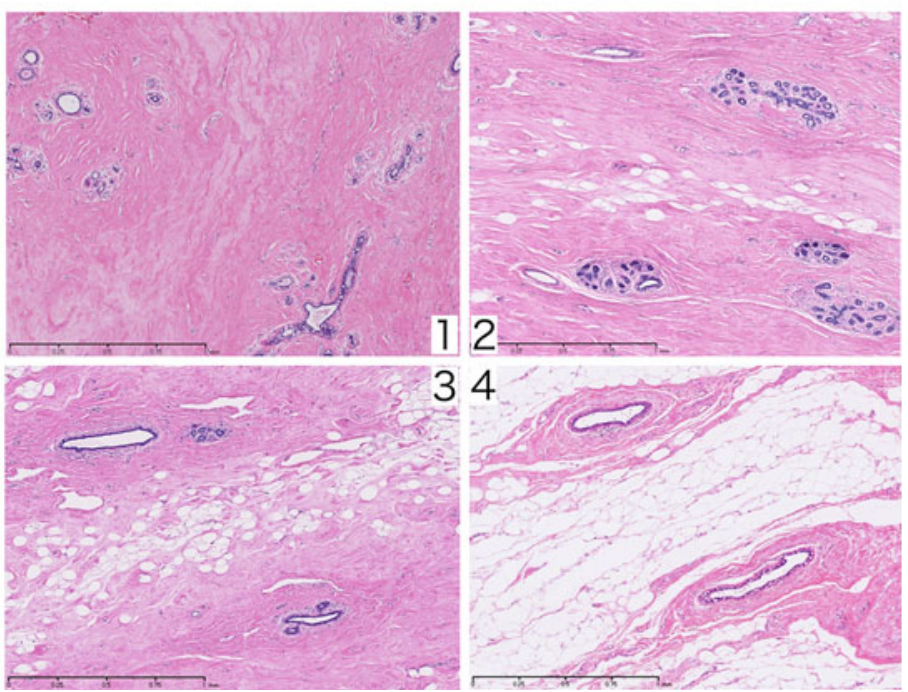

Fig. 1 a Top Stroma surrounding lobules. Bottom Stroma surrounding a duct. They are stained dark pink by hematoxylin and eosin (HE). b The space between stroma (1) is filled in by edematous stroma (2). c Left Stromata surrounding lobules and ducts are densely packed with fibrous connective tissues (1). Right The intervening stroma is edematous and loose in fibrous connective tissues (2). d Edematous stroma is replaced by fat that is arranged along the stroma. In atrophied breasts, most of the edematous stroma has been replaced by fat, with dense stroma alone remaining. e A specimen obtained by cutting out in the direction of nipple to lesion. Fat sometimes penetrates into the boundary area between the upper and lower lobes. f Small vascular run in parallel to the boundary between the lobes
(Fig. 1a-d). Stroma in the normal histological structure can be roughly divided into two types depending on the amount of fibrous connective tissues and substrate. In addition, linear structures can be observed that are definitely different from these stroma and do not contain epithelial elements.

\section{Findings in the stroma}

1. Stroma with densely packed fibrous connective tissues. The lobules (Fig. 1a, top) and ducts (Fig. 1a, bottom) are surrounded by stroma of similar appearance. Thus, the stroma in these areas is densely packed with fibrous connective tissue (Fig. 1c left), seen as dark pink in the HE staining.

2. Edematous stroma with loose fibrous connective tissues.

These stromata fill in the space between the densely packed stromal structures (1) surrounding the lobules and ducts [Fig. 1b (2)]. The substrate is edematous, and fibrous connective tissues are loose (Fig. 1c right). They are stained pale pink by HE. 

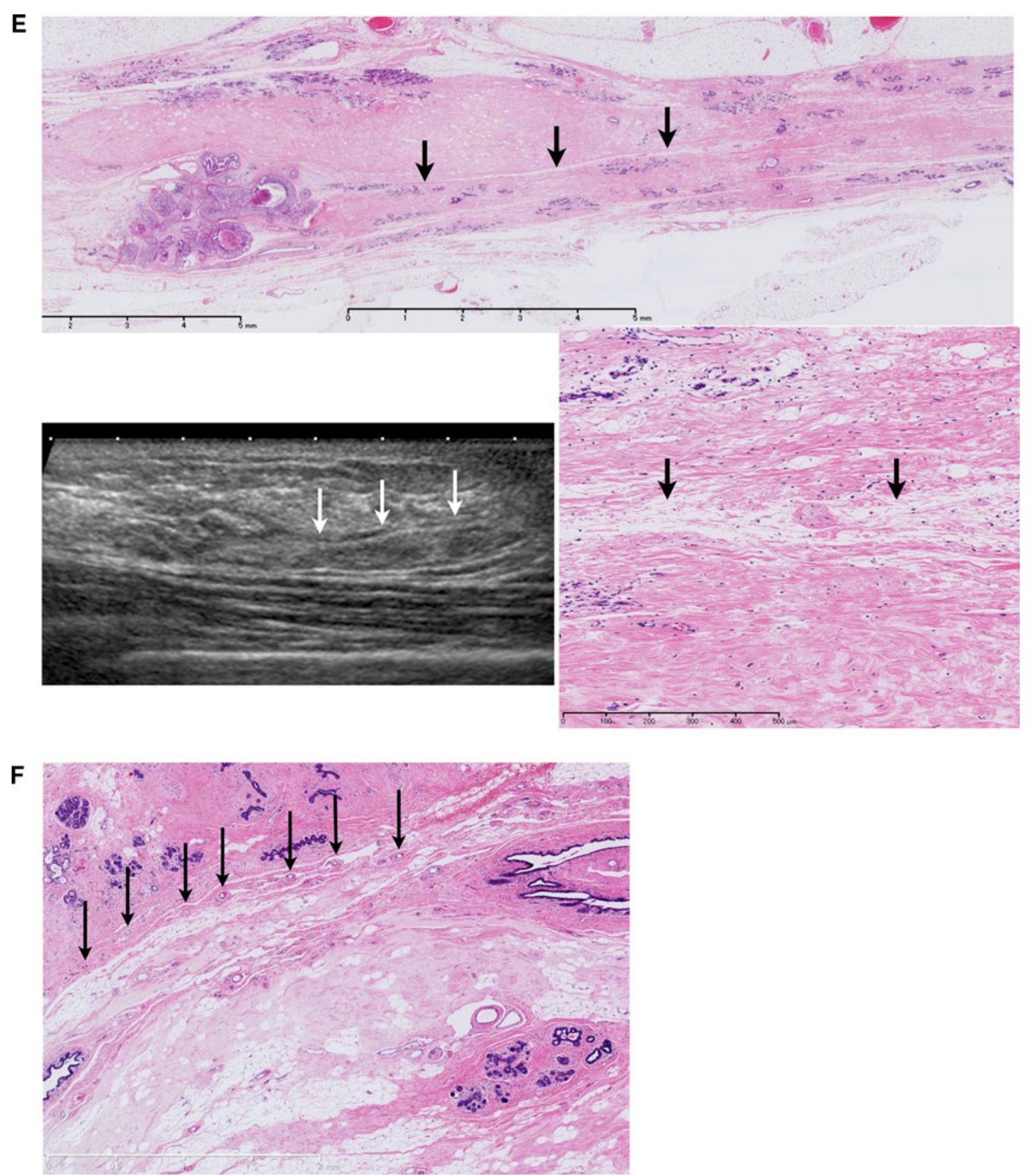

Fig. 1 continued

\section{Changes in the stroma with age}

Stroma with densely packed fibrous connective tissues (1) is observable in the breasts of all age groups, even in the highly atrophied breasts of elderly people. In contrast, stroma with loosely packed fibrous connective tissues (2) shows age-related changes. With advancing age, fat replaces stroma, thereby forming lines along the fibrous connective tissues (Fig. 1d). In highly atrophied breasts, most of the edematous stroma has been replaced by fat.

\section{Linear structures containing vessels}

Linear structures containing small vessels are observed (Fig. 1e, f). Occasionally, fat enters these structures. 
Fig. 2 (a) (1) Stroma that is densely packed with fibrous connective tissues and surround the lobules and an extralobular duct (isoechoic). (2) Edematous stroma with loosely packed fibrous connective tissues (hyperechoic). Isoechoic structures can be confirmed by ultrasound (US). The difference in acoustic impedance density between (1) and (2) is visualized as the iso- to hyperechoic area. b (1) Stroma surrounding lobules and ducts $=$ isoechoic. (2) Edematous

stroma $=$ hyperechoic. Triangle Microcyst $=$ hypoechoic.

Dotted line The area where the lobulus crowds $=$ isoechoic. c Comparison of US and the histology an age change. In stroma with loosely packed fibrous connective tissues, the ratio between edematous stroma and fat varies depending on age and other factors, but they are visualized as hyperechoic areas regardless of the ratio. $\mathbf{d}$ Lobes of various sizes overlap each other, causing varied appearance of the boundary surfaces. (1) If the lobe in front is smaller, the boundary surface is visible in the shallow area. (2) If the lobes in the front and back are of comparable size or the lobe in front is slightly larger, the boundary surface is widely visible to the terminal area. (3) If the lobe in front is larger and the lobe in back is smaller, the boundary surface is visible in the deep area

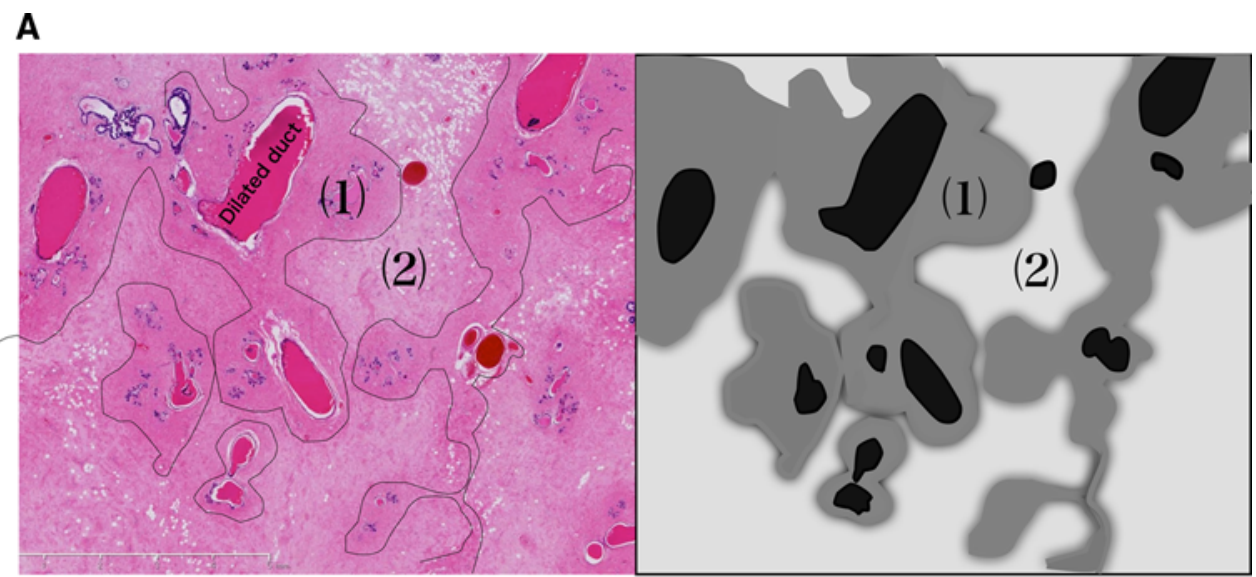

\section{B}

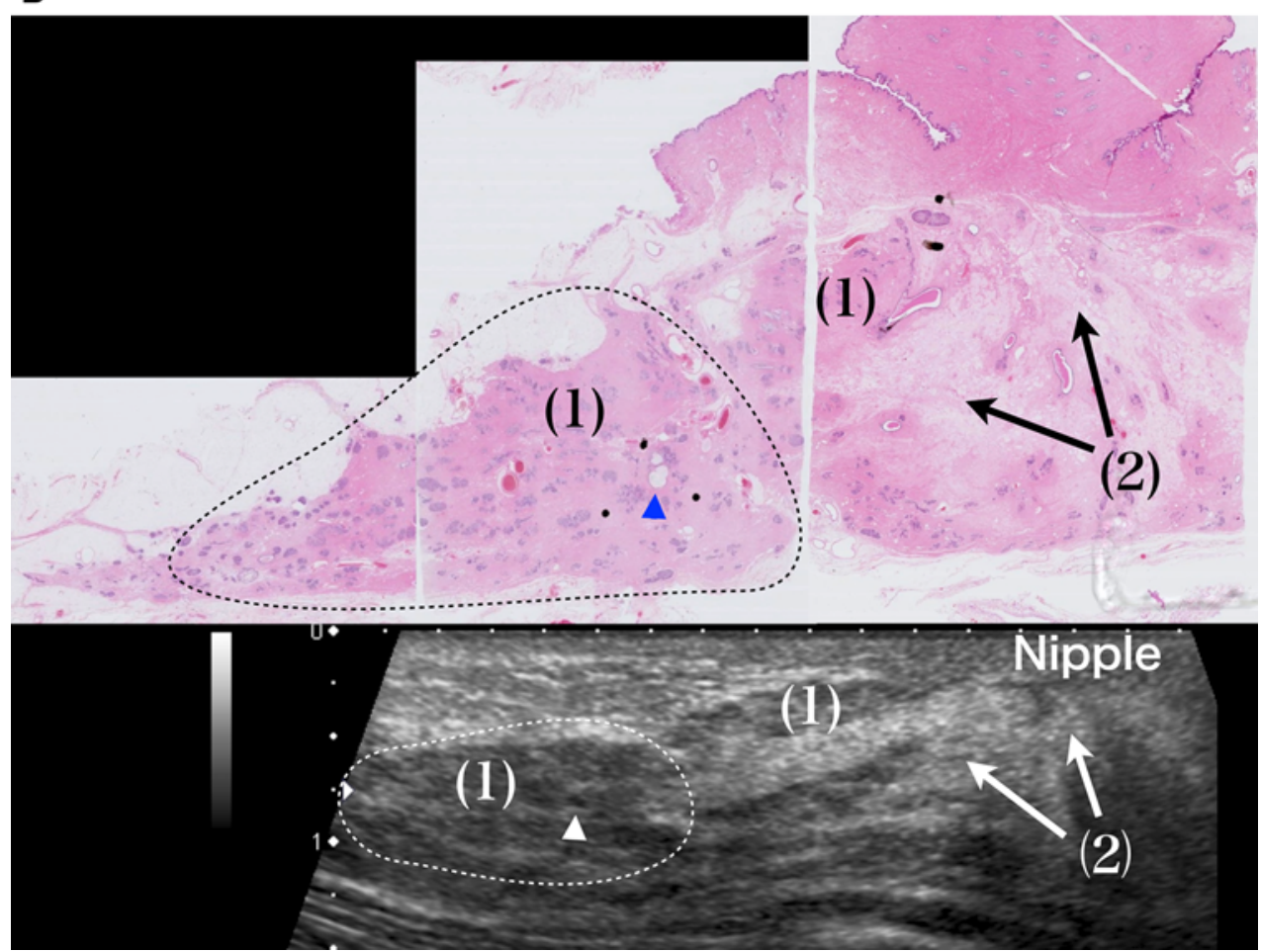

Lobules, ducts, and interstitial tissues in areas that are separated by the linear structure are confined to each area. stroma replaced by fat are visualized as hyperechoic structures.
Correlation between histological findings and US images

US images of stroma (Fig. 2a-c)

1. Stroma with densely packed fibrous connective tissues. The stroma surrounding lobules and all ducts is isoechoic to the subcutaneous fat.

2. Stroma with loosely packed fibrous connective tissues. Both edematous stroma with loosely distributed fat and
Age-related changes in stroma and US images (Fig. 2c)

Stromata with densely packed fibrous connective tissues (1) are visualized as isoechoic structures in all age groups. In stroma with loosely packed fibrous connective tissues (2), the ratio between edematous stroma and fat varies depending on age and other factors, but they are visualized as hyperechoic areas regardless of the ratio. 

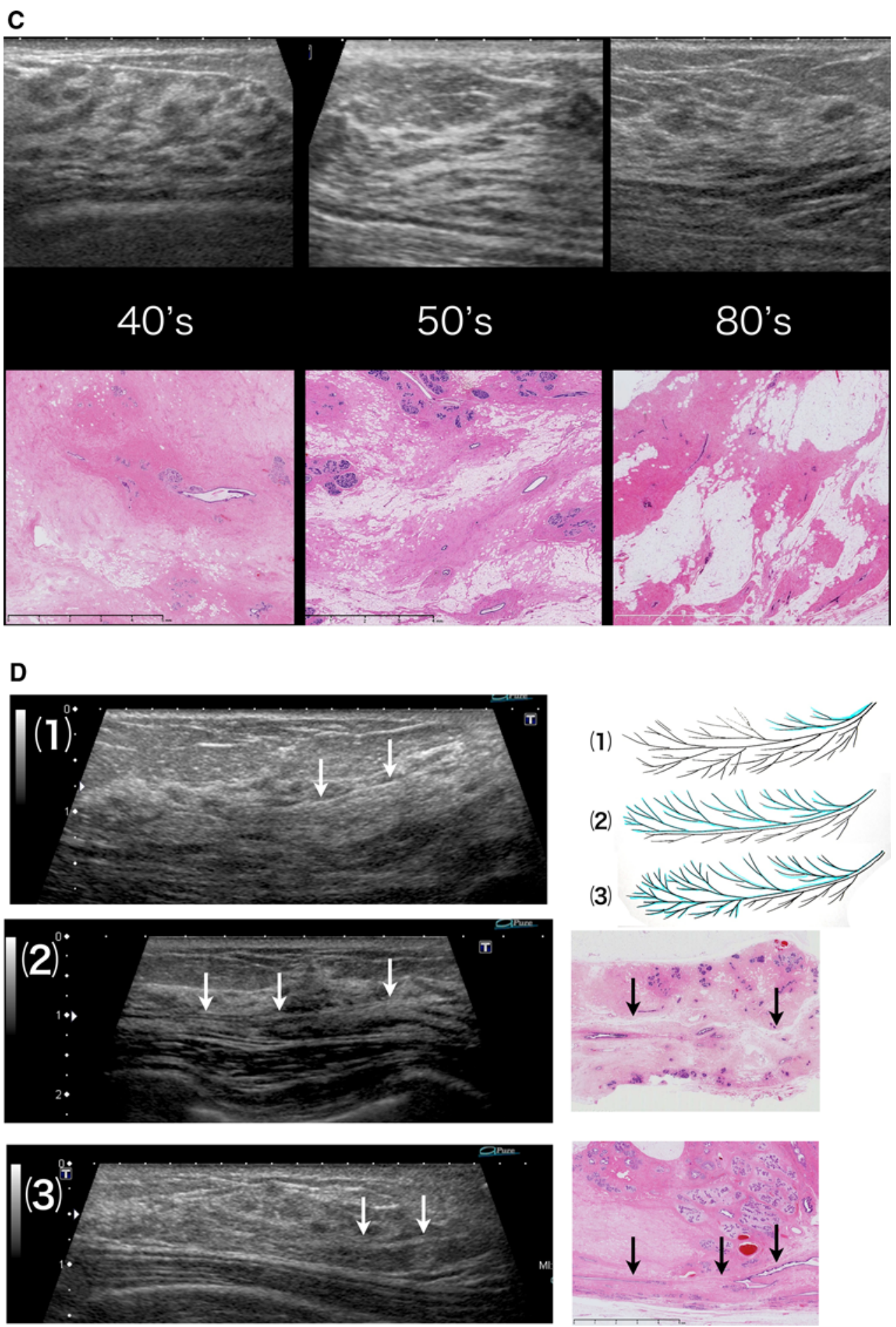

Fig. 2 continued

\section{US images of sheet structures containing vessels}

Parts of the sheet structures containing small vessels are observed as very thin structures with a "hyperechoic surface" that reflects the ultrasonic waves (Fig. 2d). If fat is present in this structure, an isoechoic pattern appears in the middle, facilitating its observation (Fig. 1e). The distribution of the isoechoic structures appears not to override the boundary surface. 


\section{Discussion}

Correlation between histological and US images, and interpretation of the results

\section{Isoechoic structures}

Comparison of the images of the histological specimens and US scans of normal breasts showed that lobules and all ducts are visualized as isoechoic structures together with the surrounding stroma with densely packed fibrous connective tissues. Thus, the isoechoic structures appear to be interrupted at some point or another, but they are structures including the surrounding stroma reflecting the course of the ducts and are observed as basic structures on US images (Fig. 3). Similarly, on images of histological specimens, the thickness of dense stroma surrounding the terminal ducts is not uniform, and some parts are very thin, which may cause the interrupting appearance of isoechoic structures. In addition, this is likely to have hindered the understanding that isoechoic structures in mammary US scans represent the courses of ducts.

The fact that these stromata with densely packed fibrous connective tissues remain unchanged with age suggests that they serve as structural support for ducts (Figs. 1d, 2c)

\section{Hyperechoic areas}

Hyperechoic areas that fill in the space between isoechoic structures are, histologically, "stromata that are rich in substrates, edematous, and contain loosely packed fibrous connective tissues." In the present study, these stromata have been confirmed to be replaced by fat with advanced age (Fig. 1d). When the proportion of edematous stroma is higher, the ultrasonic wave is reflected by diffusely scattered fibrous connective tissues and fat. In addition, when the stroma has been replaced by fat, the ultrasonic wave is reflected by the remaining fibrous connective tissues, etc. Thus, the stroma is considered to be visualized as hyperechoic areas regardless of the proportion of fat (Fig. 2c).

\section{Boundary surface between the lobes}

Consistent with the sites that are observed as sheet structures on US images, linear structures rich in vessels were identified in histological specimens (Fig. 1e, f). The ducts, lobules, and interstitial tissue in the areas on one side of the linear structure do not enter the area on the other side, which suggests that the linear structure represents the boundary between the lobes. The breast consists of mammary lobes of various sizes [5], with some of them overlapping with each other. In the present histological study, this boundary structure does not contain epithelial elements such as ducts and lobules and is rich in small vessels, which suggests that the structure observed represents the interface between one lobe in the front and the other one in the back.

On US images, parts of the boundary surface between the lobes can be identified as an extremely thin "hyperechoic sheet" structure that reflects ultrasonic waves (Fig. 1e). This structure is most clearly visible in area C, where the thickness of the mammary gland is the largest. The width and depth may differ depending on the site in the breast because of the difference in the size of the overlapping lobes (Fig. 2d).

There have been reports in which the dilated ducts or normal ducts have been traced, or in which the authors
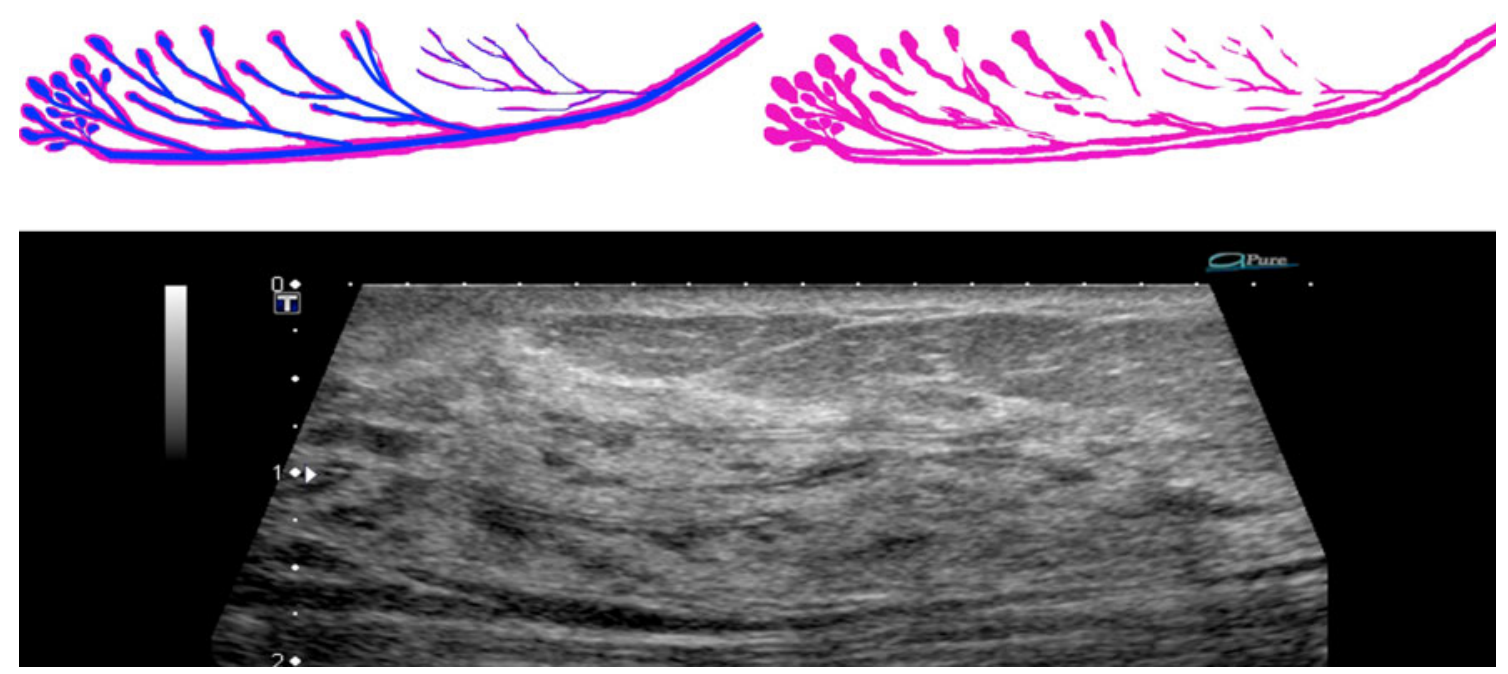

Fig. 3 Duct is straight, but stroma surrounding lobules and ducts does not form a concentric circle. The isoechoic structure observed in US is not constant in width and appears to be interrupted here and there. But they are structures including the surrounding stroma, reflecting the course of the ducts, and are observed as basic structures on US images 
Fig. 4 (1) Regularity toward the nipple. (2) Regularity toward the boundary surface of the lobes
Isoechoic structures : 2 regularities
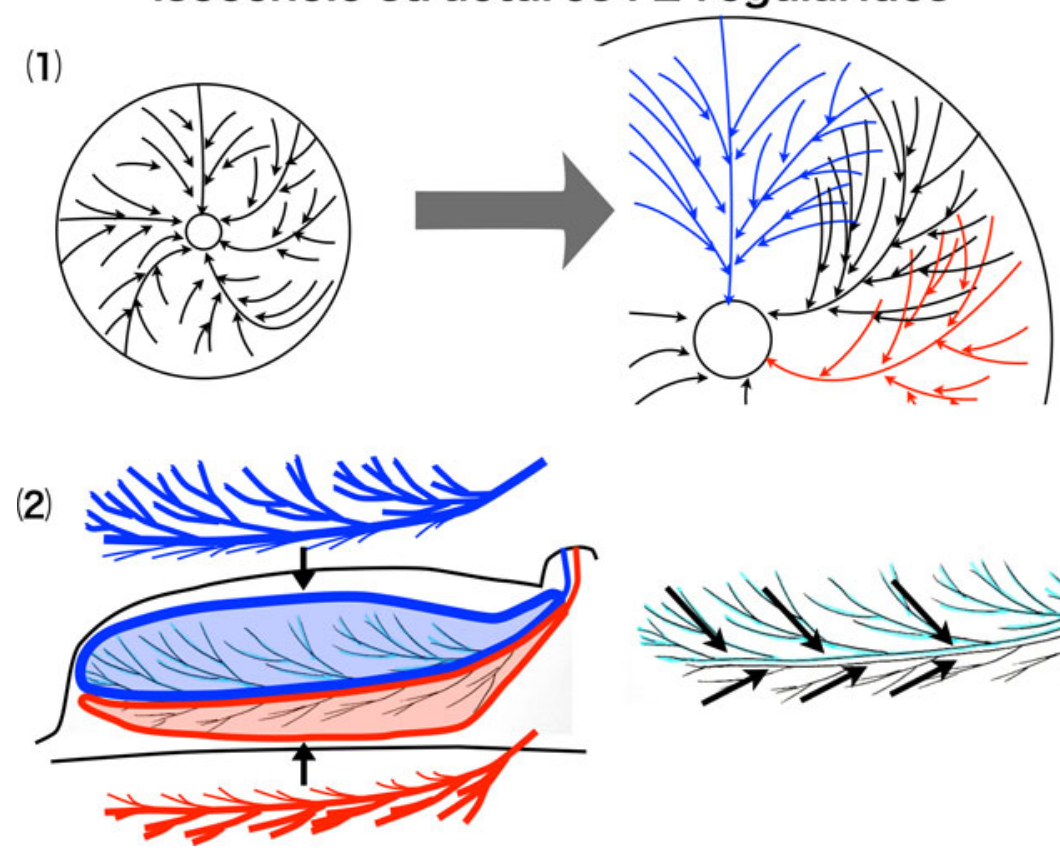

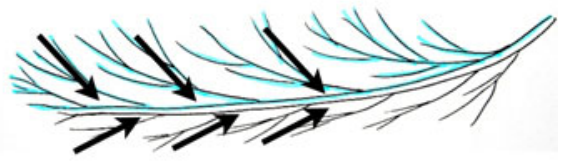

inferred the extent of the spread of the lobe on the basis of the distribution of ductal carcinoma in situ (DCIS) [3-6, 8, 9]. However, no reports have identified the boundary surface of overlapping lobes either by imaging or histology. In our method of US evaluation, one has to pay close attention to the boundary surface of the lobes when analyzing the images. Therefore, when diagnosing breast cancer using the normal structure as reference, knowledge of the three-dimensional structure of the individual lobes is essential.

Method for interpreting three-dimensional ultrasound images of mammary lobes

There are four critical points for this image reading: "regularity," “continuity," "interruption and disorder," and "uneven width." First, normal isoechoic structures have two types of regularities, i.e., (1) toward the nipple and (2) toward the boundary surface between the lobes, reflecting the course of the mammary ducts (Fig. 4). Second, the continuity of the isoechoic structure should be traced by paying attention to these two types of regularities. Third, the site where the isoechoic structure is interrupted in its continuity or disordered in direction should be evaluated for possible pathological changes. Fourth, an important point in detecting minute isoechoic lesions is noting a regional uneven width in the diameter of the isoechoic structures, which, under normal conditions, have no irregularity in the width of the diameter and are used as references.
Thus, this observational method allows the detection of minute nonmass lesions and isoechoic lesions.

The observational method that traces the continuity of the mammary ducts with the lobe in mind is thus a method of observation using the knowledge of the three-dimensional anatomy of the breast. This method of interpretation based on the knowledge of the three-dimensional anatomy of the breast is critical in the $2{ }^{\text {nd }}$ LookUS, which requires detection of the same lesion observed in other modalities.

Future challenges

The sites detected by the above-described method for further evaluation include many variations within normal ranges and benign lesions. Knowing how to identify lesions that are likely to be malignant from among these sites is important for screening for breast cancer. It will be possible to differentiate malignant from normal or benign changes by investigating the difference in the pattern of the three-dimensional internal structure or relationship with the surrounding stroma. Verification and standardization of these differentiation methods remain future challenges for us.

\section{Summary}

With the progress in diagnostic imaging, US examination is required to have a sensitivity to detect more minute malignant lesions than those detected by existing methods 
as well as malignant lesions that resemble benign lesions. In identifying these hard-to-detect lesions, anatomical knowledge of the normal structure of the breast is critical when performing US examination and is expected to contribute to its progress.

Acknowledgments Thanks are due to Dr. Akira Satoh, Department of Pathology, Takamatsu Heiwa Hospital, for his continuing guidance in the pathological discussion.

\section{References}

1. Schneck CD, Lehman DA. Sonographic anatomy of the breast. Semin Ultrasound. 1982;3:13-33.

2. Teboul M. Anatomy of the breast. In: Teboul M, Halliwell M, editors. Atlas of ductal echography of the breast: the introduction of anatomic intelligence into the breast imaging. Cambridge: Blackwell Science; 1995. p. 49-82.

3. Ramsay DT, Kent JC, Hartmann RA, Hartmann PE. Anatomy of the lactating human breast redefined with ultrasound imaging. J Anat. 2005;206:525-34.
4. Gooding MJ, Finlay J, Shipley JA, Halliwell M, Duck FA. Threedimensional ultrasound imaging of mammary ducts in lactating women. J Ultrasound Med. 2010;29:95-103.

5. Going JJ, Moffat DF. Escaping from Flatland: clinical and biological aspects of human mammary duct anatomy in three dimensions. J Pathol. 2004;203:538-44.

6. Ohtake T, Kimijima I, Fukushima T, Yasuda M, Sekikawa K, Takenoshita $\mathrm{S}$, et al. Computer-assisted complete three-dimensional reconstruction of the mammary ductal/lobular systems: implications of ductal anastomoses for breast-conserving surgery. Cancer. 2001;91:2263-72.

7. Stavros AT. Breast anatomy: the basis for understanding sonography. In: McAllister L, Donnellan K, Martin SP, Rothschild R, editors. Breast ultrasound. Philadelphia: Lippincott Williams \& Wilkins; 2004. p. 56-108.

8. Teboul M, Halliwell M. Atlas of ultrasound and ductal echography of the breast: the introduction of anatomic intelligence into breast imaging. Oxford: Blackwell Science; 1995.

9. Going JJ, Mohun TJ. Human breast duct anatomy, the 'sick lobe' hypothesis and intraductal approaches to breast cancer. Breast Cancer Res Treat. 2006;97:285-91. 